\title{
Avaliação de Oligochaeta (Tubificinae) e Polychaeta (Namalycastis abiuma) como bioindicadores da composição isotópica de Chumbo: exemplo do estuário guajari- no, Belém (PA), Brasil
}

\author{
Simone Pereira de OLIVEIRA ${ }^{1}$, Candido Augusto Veloso MOURA² \& José Souto de ROSA FILHO ${ }^{3}$ \\ 1. Instituto Federal de Educação, Ciência e Tecnologia do Pará, Campus Conceição do Araguaia. Av. Couto Magalhães, CEP 68540-000, Con- \\ ceição do Araguaia, PA, Brasil. E-mail: simone.oliveira@ifpa.edu.br. \\ 2. Instituto de Geociências, Universidade Federal do Pará. Caixa Posta 8608, CEP 66075-110, Belém, PA, Brasil. E-mail: candido@ufpa.br. \\ 3. Laboratório de Bentos, DOCEAN/CTG, Universidade Federal de Pernambuco. Av. Prof. Moraes Rego, S/N, CEP 50670-901, Recife, PE, \\ Brasil.E-mail: souto.rosa@ufpe.br.
}

Recebido em 02/2011. Aceito para publicação em 06/2013.

Versão online publicada em xx/xx/20xx (www.pesquisasemgeociencias.ufrgs.br)

\begin{abstract}
Resumo - A composição isotópica de chumbo foi determinada em amostras de organismos bentônicos, representantes das classes Oligochaeta e Polichaeta, e sedimentos de fundo, ambos coletados na baía do Guajará (Miramar e canal do Una) e rio Guamá (Igarapé Tucunduba), objetivando comparar a assinatura isotópica do elemento nos organismos e nos sedimentos, e identificar a adequabilidade desses organismos como bioindicadores da contaminação dos sedimentos por esse metal. Desse modo, foi empregado um procedimento que consistiu em dividir os valores das razões ${ }^{206} \mathrm{~Pb} /{ }^{204} \mathrm{~Pb},{ }^{207} \mathrm{~Pb} /{ }^{204} \mathrm{~Pb},{ }^{208} \mathrm{~Pb} /{ }^{204} \mathrm{~Pb}$ e ${ }^{207} \mathrm{~Pb} /{ }^{206} \mathrm{~Pb}$ determinados nos organismos, pelos valores dessas razões encontradas nos sedimentos. Para o quociente resultante (R) foi adotado o valor de tolerância de $1 \pm 0,004(0,996 \leq \mathrm{R} \leq 1,004)$ para indicar a similaridade entre as composições isotópicas do chumbo dos organismos e do sedimento de fundo. Em $75 \%$ das amostras biológicas os valores de $\mathrm{R}(0,997 \leq \mathrm{R} \leq 1,003)$ ficaram no intervalo aceitável. Apenas as amostras de Oligoquetas coletadas próximo à desembocadura do canal Una apresentaram valores fora do intervalo indicado $(1,001 \leq R \leq 1,017)$. Esse desvio pode ser atribuído a uma contribuição antropogênica recente no local de coleta, registrado pela presença de emulsão asfáltica na porção superficial do sedimento. Nessas condições, sugere-se que os organismos analisados não registram mudanças súbitas na composição isotópica do chumbo em seu habitat devido, provavelmente, ao lento processo de acumulação do metal em seus tecidos. No entanto, os dados confirmam a hipótese de que esses organismos são bons bioindicadores, podendo ser aplicados como uma matriz alternativa em estudos ambientais com isótopos de chumbo.
\end{abstract}

Palavras-chave: sedimento de fundo, isótopos de chumbo, rio Guamá, baía do Guajará, bioindicador.

Abstract - Assessment of Oligochaeta (Tubificinae) and Polychaeta (Namalycastis abiuma) as bioindiCATORS OF THE LEAD ISOTOPE COMPOSITION IN ITS HABITAT: EXAMPLE OF THE GUAJARÁ ESTUARY, BELÉM (PA), BRAzIL. This study reports lead isotope data from benthic organisms (representatives of the classes Oligochaeta and Polichaeta) and bottom sediments samples, which were collected in the Guajara bay (Miramar and Una channel) and Guama river (Tucunduba Creek) in order to investigate if these organisms could be used as bioindicators in environmental studies. The comparison between the lead isotope signature of the benthic organisms and that of the bottom sediments of their habitat was performed by dividing the values of the ${ }^{206} \mathrm{~Pb} /$ ${ }^{204} \mathrm{~Pb},{ }^{207} \mathrm{~Pb} /{ }^{204} \mathrm{~Pb},{ }^{208} \mathrm{~Pb} /{ }^{204} \mathrm{~Pb}$ and ${ }^{206} \mathrm{~Pb} /{ }^{207} \mathrm{~Pb}$ ratios determined in the organisms by the values of these ratios found in the sediments. When the value of the calculated quotient $(R)$ was $1 \pm 0.004(0.996 \leq R \leq 1.004)$ the lead isotope compositions of the benthic organisms and that of the bottom sediment were considered similar. The values of $\mathrm{R}$ for $75 \%$ of the biological samples were in this range $(0.997 \leq \mathrm{R} \leq 1.003)$. Only the Oligochaeta collected nearby the Una channel presented a deviation of the interval(1.001 $\leq \mathrm{R} \leq 1.017)$. This difference was related to a recent anthropogenic contribution in this area, recorded by the presence of asphalt emulsion in the top portion of the sediment. Under these conditions, it is suggested that the analyzed organisms do not record sudden lead isotopes changes in their habitat, probably due to the slow accumulation of the metal in their tissues. However, the data confirm the hypothesis that these organisms are good bioindicators and can be applied as an alternative matrix to environmental studies using lead isotopes.

Keywords: bottom sediment, lead isotope, Guajara bay, Guama River, bioindicator. 


\section{Introdução}

No meio ambiente, elementos químicos como o chumbo tem sua composição isotópica modificada por causas naturais e antrópicas. As razões ${ }^{206} \mathrm{~Pb} /{ }^{204} \mathrm{~Pb}$, ${ }^{207} \mathrm{~Pb} /{ }^{204} \mathrm{~Pb}$ e ${ }^{208} \mathrm{~Pb} /{ }^{204} \mathrm{~Pb}$ podem indicar contribuição antropogênica, caso a composição isotópica observada seja distinta daquela de origem geogênica. A aplicação da razão isotópica para diagnósticos ambientais de áreas suscetíveis a poluição por chumbo é mais eficaz do que utilizar a concentração do metal. Isso se deve ao fato de modificações na composição isotópica do chumbo poder ser detectadas antes das concentrações do metal atingir níveis críticos (Moura et al., 2004). Dado essa vantagem, estudos empregando isótopos de chumbo têm sido realizados em diversos materiais, como solo, sedimento, sangue, vegetais e animais (Chaudary-Webb et al., 2003; Scheuhammer et al., 2003; Notten et al., 2008).

Em sistemas aquáticos contaminados, uma pequena parte do chumbo está dissolvida na água, pois maior parte do metal encontra-se fortemente ligada à fração fina do sedimento, onde o chumbo pode ser mobilizado geoquimicamente pelas mudanças físico-químicas do substrato e consumidos pelos organismos (Azevedo \& Chasin, 2003). Em função disso, Oligochaeta e Polychaeta têm sido utilizados como bioindicadores de contaminação ambiental por metais pesados (Langston, 1986; Pullianen et al. 1986; Clark, 2001; Singh et al., 2007), fato atribuído à grande capacidade de bioacumulação de metais por esses anelídeos bentônicos (Pullianen et al., 1986; Gray et al., 1992; Singh et al., 2007) e sua ampla distribuição geográfica (Fauchald \& Jumars, 1979; Giere \& Pfannkuche, 1982).

Apesar de sua adequação como indicador biológico de contaminação ambiental por metais pesados, existe um número reduzido de estudos voltados para a determinação da composição isotópica do chumbo em anelídeos. Scheuhammer et al. (2003) estudando a composição isotópica do chumbo nos oligoquetas Aporrectodea tuberculata (Eisen, 1874) e Lumbricus rubellus (Hoffmeister, 1843), para investigar a contaminação na ave migratória Scolopax minor (Gmelin, 1789), perceberam que pássaros com concentração normal de chumbo (<20 mg.kg-1) possuíam a mesma composição isotópica dos Oligochaetas terrestres dos quais se alimentavam.

Pesquisas empregando razão isotópica do chumbo em diferentes ambientes têm demonstrado a eficiência dessa ferramenta em estudos ambientais. Carigan \& Geriépy (1995) utilizaram a composição isotópica do chumbo em liquens epifíticos e sedimentos lacustres como um traçador de fontes de emissões atmosféricas de chumbo. Como resultado, perceberam que os liquens registravam a presença de chumbo, num raio de até $500 \mathrm{~km}$ da fonte de emissão. Através da análise da composição isotópica de chumbo no sangue em mulheres da Cidade do México, Chaudary-Webb et al.
(2003) constataram que estas estavam sendo contaminadas por utensílios domésticos produzidos de cerâmica. Gioia et al. (2006) identificaram a descarga de esgoto tratado como importante fonte de contaminação de chumbo no lago Paranoá em Brasília (DF), através do uso de isótopos deste metal em sedimentos de fundo.

Na região metropolitana de Belém (PA) a drenagem natural, que possui cerca de $30 \mathrm{~km}$ de extensão (COHAB, 1997), transporta os efluentes domésticos e industriais para a baia do Guajará (Nascimento, 1995). Diariamente são produzidos na região cerca de 274,5 milhões de litros de esgotos, sendo $8 \%$ coletados, e destes não mais que $3 \%$ são tratados antes de ser lançado diretamente nos corpos de água e no solo (IBGE, 2008). Diversos estudos têm demonstrado que o lançamento de efluentes tem causado perda da qualidade da água e nos sedimentos na baía do Guajará e rio no Guamá. Em locais próximos a fontes de lançamentos têm sido registradas concentrações elevadas de coliformes fecais, óleos e graxas, e condições hipóxicas da água (Lima \& Santos, 2001; Ramos, 2004). Altas concentrações de poluentes metálicos nos sedimentos também foram detectadas por Corrêa \& Pereira (2002). Viana et al. (2009) e Aviz et al. (2012) identificaram alterações nas comunidades de peixes e de macroinvertebrados bentônicos, os quais apresentaram maiores densidades de organismos nas áreas próximas aos principais pontos de lançamento de efluentes urbanos.

Na região metropolitana de Belém (PA), Moura et al. (2004) e Nascimento (2007) caracterizaram a assinatura isotópica do chumbo atual em sedimentos de fundo do sistema hidrográfico. Sugeriram que os valores em torno de 1,20 da razão ${ }^{206} \mathrm{~Pb} /{ }^{207} \mathrm{~Pb}$ são originados de fontes geogênicas, enquanto as assinaturas isotópicas de fontes antropogênicas apresentam valores da razão ${ }^{206} \mathrm{~Pb} /{ }^{207} \mathrm{~Pb}$ em torno de 1,160 .

0 presente estudo determinou a composição isotópica do chumbo em oligoquetas Tubificinae e no poliqueta Namalycastis abiuma Grube, 1872 e nos sedimentos de fundo na baia do Guajará e rio Guamá (Região Metropolitana de Belém, PA), com o objetivo de avaliar a aplicabilidade destes organismos como uma matriz alternativa para estudos ambientais utilizando isótopos de chumbo.

\section{2 Área de estudo}

A baía de Guajará e o rio Guamá (Fig. 1) fazem parte do estuário Guajará, inserido no estuário amazônico, que possui circulação uniforme, com tendência a homogeneidade vertical dos parâmetros físicos e químicos da água entre a preamar e baixa-mar (Pinheiro, 1987). 0 aporte de água doce, provinda principalmente dos rios Amazonas e Pará, praticamente evita a entrada de água salgada do oceano adjacente, fazendo com que a água da baía de Guajará tenha ao longo de todo o ano características oligohalinas, com salinidade entre 0-10 \%o (Aviz et al., 2012). As marés são semi-diurnas com 
amplitude máxima de 2-3 m e correntes superficiais que alcançam 1,9-2,2 m.s ${ }^{-1}$. Os sedimentos de fundo são lamosos (cerca de 70 \% da área) e arenosos, e ocorrem em bancos submersos (Gregório \& Mendes, 2009).

Nessa região, o clima é tropical úmido com temperatura media do ar variando de 24 a $32{ }^{\circ} \mathrm{C}$, sempre su- perior a $18^{\circ} \mathrm{C}$. Os padrões de precipitação pluviométrica definem dois períodos climáticos, um chuvoso, que se estende de janeiro a junho com precipitação mensal média de $1657 \mathrm{~mm}$; e outro seco, de julho a dezembro, com precipitação média mensal de $487 \mathrm{~mm}$ (Moraes et al., 2005).

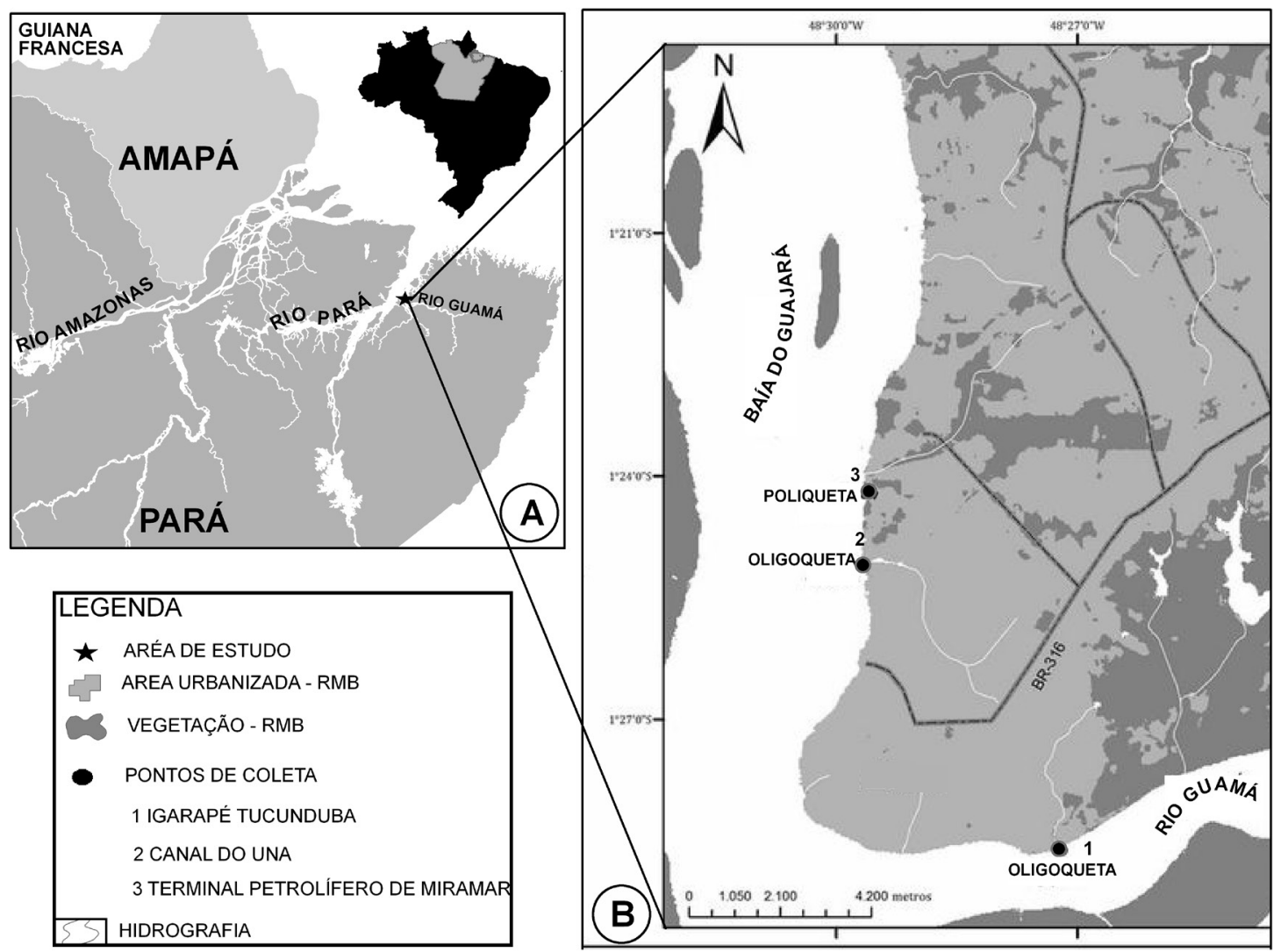

Figura 1. Localização da área de estudo: (A) Mapa simplificado do estuário amazônico indicando a área de estudo. (B) Indicação dos pontos de coleta na Região Metropolitana de Belém (RMB).

\section{Materiais e métodos}

\subsection{Procedimentos de campo}

As coletas de organismos e de sedimento de fundo ocorreram na foz do igarapé Tucunduba no rio Guamá (Ponto 1), no porto de Miramar (Ponto 2) e no canal do Una (Ponto 3), ambos na baía do Guajará. Dado que Namalycastis abiuma e os Tubificinae têm ciclo de vida longo (mais de seis meses), se optou por realizar apenas uma amostragem, desconsiderando mudanças temporais nas características da água. No canal do Una e no igarapé Tucunduba foram coletadas amostras de Tubificinae, e em Miramar de N. abiuma, com pegador de fundo tipo van Veen, sendo realizadas por local, oito pegadas para amostras de organismos e uma pegada para sedimento.

\subsection{Procedimentos de laboratório}

As amostras biológicas foram passadas em malha de 0,3 mm de abertura, sendo os oligoquetas e polique- tas separados. Buscou-se separar organismos de tamanhos similares, que teriam idades semelhantes. Estes foram liofilizados por $24 \mathrm{~h}$ (liofilizador tipo Edwards). Após a liofilização, massas de 100-500 mg de amostra foram atacadas com $\mathrm{HNO}_{3}$ (tridestilado) $5 \mathrm{~N}$ e aquecidas em chapa a $100^{\circ} \mathrm{C}$ por $3 \mathrm{~h}$ para a dissolução. Depois da abertura, as amostras foram evaporadas e aos resíduos secos foram adicionados $5 \mathrm{ml}$ de $\mathrm{HBr}$ (tridestilado) $5 \mathrm{~N}$ e novamente evaporados à $100^{\circ} \mathrm{C}$.

Uma alíquota de $40 \mathrm{~g}$ de sedimento foi seca em estufa por $24 \mathrm{~h}$, macerada em gral de ágata, e passada em peneira de $63 \mu \mathrm{m}$ para a separação da fração fina, utilizada na análise. Para a lixiviação destas amostras foi utilizado $\mathrm{HNO}_{3}$ (tridestilado) $5 \mathrm{~N}$. As soluções lixiviadas foram aquecidas em chapa a $100{ }^{\circ} \mathrm{C}$ por cerca de $3 \mathrm{~h}$. Aos resíduos secos remanescentes foram adicionados $5 \mathrm{ml}$ de $\mathrm{HBr}$ (tridestilado) $8 \mathrm{~N}$ e posteriormente evaporados a $100{ }^{\circ} \mathrm{C}$.

Para a separação do chumbo biodisponível nos organismos e sedimentos foi empregado o método descrito por Rodrigues (1992). A coluna de teflon com 20 $\mathrm{mm}$ de altura e $4 \mathrm{~mm}$ de diâmetro foi preenchida com resina (50-60 $\mu$ l) tipo DOWEX AG 1 x 8, 240-400 mesh. 
A separação do chumbo ocorreu utilizando dois ácidos ( $\mathrm{HCl} 6 \mathrm{~N}, \mathrm{HBr} 0,5 \mathrm{~N}$ ) e água Milli-Q. Para a aglutinação do chumbo separado foi usado $\mathrm{H}_{2} \mathrm{PO}_{3} 0,125 \mathrm{~N}$. Os resíduos obtidos da separação foram misturados à sílica gel, e então depositados em filamento de rênio. A determinação da composição isotópica do chumbo foi feita no espectrômetro de massa de Termoionização (modelo FINNIGAN MAT 262) do Laboratório de Geologia Isotópica da UFPA.

\subsection{Análise dos dados}

Para os sedimentos e organismos foram calculadas as razões ${ }^{206} \mathrm{~Pb} /{ }^{204} \mathrm{~Pb},{ }^{207} \mathrm{~Pb} /{ }^{204} \mathrm{~Pb}$ e ${ }^{208} \mathrm{~Pb} /{ }^{204} \mathrm{~Pb}$. Para a análise ambiental foram considerados apenas os valores da razão ${ }^{206} \mathrm{~Pb} /{ }^{207} \mathrm{~Pb}$, prática comum em estudos ambientais com isótopos de chumbo (Carvalho, 2001; Scheuhammer et al., 2003; Nascimento, 2007).

As composições isotópicas do chumbo nos organismos e no sedimento foram comparadas visando identificar a capacidade dos organismos de refletir a composição isotópica desse metal no ambiente. Essa comparação foi realizada dividindo os valores das razões ${ }^{206} \mathrm{~Pb} /{ }^{204} \mathrm{~Pb},{ }^{207} \mathrm{~Pb} /{ }^{204} \mathrm{~Pb},{ }^{208} \mathrm{~Pb} /{ }^{204} \mathrm{~Pb}$ e ${ }^{206} \mathrm{~Pb} /{ }^{207} \mathrm{~Pb}$ dos organismos pelos valores dessas razões nos sedimentos dos mesmos locais de coleta. 0 quociente dessa divisão, representado por R, é igual a 1 caso haja total similaridade. Para efeito dessa comparação adotou-se o intervalo entre 0,996 e 1,004 $(0,996 \leq R \leq 1,004)$, como indicativo de que as razões isotópicas de chumbo dos organismos eram similares àquelas do ambiente (Chaudhary-Webb et al., 2003).

\section{Resultados}

Os valores das razões ${ }^{206} \mathrm{~Pb} /{ }^{204} \mathrm{~Pb},{ }^{207} \mathrm{~Pb} /{ }^{204} \mathrm{~Pb}$,
${ }^{208} \mathrm{~Pb} /{ }^{204} \mathrm{~Pb}$ e ${ }^{206} \mathrm{~Pb} /{ }^{207} \mathrm{~Pb}$ nos organismos e sedimentos são apresentados na tabela 1. A análise dos valores da razão ${ }^{206} \mathrm{~Pb} /{ }^{207} \mathrm{~Pb}$ permite observar que existem diferenças na composição isotópica do chumbo nos pontos amostrados. Na foz do igarapé Tucunduba (Ponto 1), o valor da razão ${ }^{206} \mathrm{~Pb} /{ }^{207} \mathrm{~Pb}$ no sedimento foi de 1,193 e as amostras de Oligoquetas apresentaram valores entre 1,189 e 1,193. Na desembocadura do canal do Una (Ponto 2), o sedimento mostrou valor da razão ${ }^{206} \mathrm{~Pb} /{ }^{207} \mathrm{~Pb}$ de 1,167 e as amostras de Oligoquetas apresentaram valores de 1,183 e 1,184. No Terminal Petrolífero de Miramar (Ponto 3), onde foi coletado $\mathrm{Na}$ malycastis abiuma, o valor da razão ${ }^{206} \mathrm{~Pb} /{ }^{207} \mathrm{~Pb}$ no sedimento de fundo foi de 1,188, enquanto nas amostras de poliquetas variou entre 1,185 e 1,189.

Na tabela 1 estão os valores de R para ${ }^{206} \mathrm{~Pb} /{ }^{204} \mathrm{~Pb}$, ${ }^{207} \mathrm{~Pb} /{ }^{204} \mathrm{~Pb},{ }^{208} \mathrm{~Pb} /{ }^{204} \mathrm{~Pb}$ e ${ }^{206} \mathrm{~Pb} /{ }^{207} \mathrm{~Pb}$, todos estão situados entre 0,997 e 1,017. Na foz do igarapé Tucunduba os valores de R se situaram entre 0,997 e 1,001. No porto Miramar esses valores variaram entre 0,997 e 1,003. Na desembocadura do canal do Una os valores de $\mathrm{R}$ variaram de 1,001 a 1,017. Neste local, foram observados os valores mais altos de $\mathrm{R}$ para as razões ${ }^{206} \mathrm{~Pb} /{ }^{204} \mathrm{~Pb}$, ${ }^{208} \mathrm{~Pb} /{ }^{204} \mathrm{~Pb}{ }^{206} \mathrm{~Pb} /{ }^{207} \mathrm{~Pb}(1,015$ a 1,017).

\section{Discussão}

Na foz do igarapé Tucunduba (Ponto 1), situada na margem direita do rio Guamá, os valores de R estão dentro do intervalo de tolerância definido para uma associação positiva $(0,996 \leq R \leq 1,004)$ entre os organismos e o sedimento, indicando que os oligoquetas incorporaram a assinatura isotópica do chumbo de seu habitat. Nos arredores deste ponto de coleta predominam três situações que explicam seu comprometimento ambiental: 1) na margem direita do rio Guamá,

Tabela 1. Valores das razões isotópicas do chumbo dos organismos (ORG) Oligochaeta (O), Polychaeta (P), Sedimentos (SED) e o quociente (R) das razões isotópicas de chumbo determinadas nos organismos e sedimentos. Valores de R compreendido entre 0,996 e 1,004 indicam a similaridade entre os valores da razão isotópica dos organismos e sedimentos.

\begin{tabular}{|c|c|c|c|c|c|c|c|c|c|c|c|c|c|}
\hline \multirow[b]{2}{*}{ PONTOS } & \multirow[b]{2}{*}{ ID } & \multicolumn{3}{|c|}{${ }^{206} \mathrm{~Pb} /{ }^{204} \mathrm{~Pb}$} & \multicolumn{3}{|c|}{${ }^{207} \mathrm{~Pb} /{ }^{204} \mathrm{~Pb}$} & \multicolumn{3}{|c|}{${ }^{208} \mathrm{~Pb} /{ }^{204} \mathrm{~Pb}$} & \multicolumn{3}{|c|}{${ }^{206} \mathrm{~Pb}^{/ 207} \mathrm{~Pb}$} \\
\hline & & ORG & SED & $\mathrm{R}_{1}$ & ORG & SED & $\mathrm{R}_{2}$ & ORG & SED & $\mathrm{R}_{3}$ & ORG & SED & $\mathrm{R}_{4}$ \\
\hline \multirow{3}{*}{1} & 01-1 & 18,67 & 18,70 & 0,998 & 15,69 & 15,68 & 1,001 & 38,79 & 38,80 & 1,000 & 1,190 & 1,193 & 0,998 \\
\hline & $01-2$ & 18,66 & 18,70 & 0,998 & 15,69 & 15,68 & 1,001 & 38,77 & 38,80 & 0,999 & 1,189 & 1,193 & 0,997 \\
\hline & $01-3$ & 18,70 & 18,70 & 1,000 & 15,68 & 15,68 & 1,000 & 38,81 & 38,80 & 1,000 & 1,193 & 1,193 & 1,000 \\
\hline & $02-1$ & 18,54 & 18,23 & 1,017 & 15,66 & 15,63 & 1,002 & 38,60 & 38,16 & 1,012 & 1,184 & 1,167 & 1,015 \\
\hline & $02-2$ & 18,52 & 18,23 & 1,016 & 15,65 & 15,63 & 1,001 & 38,52 & 38,16 & 1,009 & 1,183 & 1,167 & 1,015 \\
\hline & P3-1 & 18,55 & 18,60 & 0,997 & 15,65 & 15,65 & 1,000 & 38,57 & 38,64 & 0,998 & 1,185 & 1,188 & 0,997 \\
\hline \multirow[t]{2}{*}{3} & P3-2 & 18,59 & 18,60 & 0,999 & 15,65 & 15,65 & 1,000 & 38,63 & 38,64 & 1,000 & 1,188 & 1,188 & 0,999 \\
\hline & P3-3 & 18,63 & 18,60 & 1,002 & 15,67 & 15,65 & 1,001 & 38,74 & 38,64 & 1,003 & 1,189 & 1,188 & 1,000 \\
\hline
\end{tabular}


em direção à montante da desembocadura do igarapé Tucunduba, o rio é protegido por mata ciliar e aparenta estar melhor preservado; 2) ainda na margem direita, à jusante da desembocadura do igarapé, não existe mata ciliar e as margens são ocupadas por edificações, nas quais são desenvolvidos variados tipos de atividades; 3) o igarapé Tucunduba tem suas margens povoadas e boa parte dos resíduos sólidos e efluentes domésticos produzidos pela população são lançados na água. Aviz et al. (2012) apontam o igarapé Tucunduba como uma das áreas do estuário Guajará com mais sintomas de impacto ambiental, tendo registrado neste local elevada abundância e dominância de oligoquetas indicadores de enriquecimento orgânico dos sedimentos.

No Terminal Petrolífero de Miramar (Ponto 3) os valores de $\mathrm{R}$ situaram-se dentro do intervalo de tolerância definido, revelando que Namalycastis abiuma também incorporou a assinatura isotópica do chumbo do habitat. Nesta área ocorrem estocagem e armazenamento de derivados de petróleo e Corrêa \& Pereira (2002) identificaram, nos sedimentos de fundo, concentrações de metais pesados acima dos limites aceitos pela legislação brasileira. Segundo esses autores, ainda que uma parte dos metais no sedimento seja de origem natural, o escoamento superficial da Região Metropolitana de Belém e o despejo direto de efluentes domésticos e industriais na baía do Guajará causam enriquecimento dos sedimentos com poluentes metálicos.

$\mathrm{Na}$ desembocadura do canal do Una (Ponto 2) os valores de $\mathrm{R}$ das razões ${ }^{206} \mathrm{~Pb} /{ }^{204} \mathrm{~Pb},{ }^{208} \mathrm{~Pb} /{ }^{204} \mathrm{~Pb}$ ${ }^{206} \mathrm{~Pb} /{ }^{207} \mathrm{~Pb}$ variaram entre 1,015 e 1,017 , o que indica que os Oligochaeta deste ponto não incorporaram plenamente a composição isotópica do chumbo do substrato. Este canal drena grande volume de esgotos domésticos da cidade de Belém e os lança, sem tratamento, na baía do Guajará. Neste local, Ramos (2004) e Aviz et al. (2012) registraram condições de hipóxia da água e enriquecimento orgânico dos sedimentos, mostrando o forte impacto ambiental na baia do Guajará na proximidade do canal do Una.

Essa influência antropogênica é observada no valor da razão ${ }^{206} \mathrm{~Pb} /{ }^{207} \mathrm{~Pb}$ de 1,16 dos sedimentos de fundo na desembocadura do canal do Una, que é mais baixo que os valores determinados para a baía do Guajará $(1,188$ e 1,172$)$ por Nascimento (2007). No entanto, os valores da razão ${ }^{206} \mathrm{~Pb} /{ }^{207} \mathrm{~Pb}$ nos oligoquetas da desembocadura do canal do Una são mais próximos daqueles encontrados para a baia do Guajará do que o valor registrado no seu habitat. Uma provável explicação para essa diferença é que a entrada recente e intensa de efluentes municipais contendo resíduos de emulsão asfáltica pode ter provocado, no momento da coleta, uma mistura de chumbo proveniente do sedimento com o da emulsão, possivelmente presente na porção superficial do sedimento. Embora não se conheça a composição isotópica do chumbo no asfalto, Lima et al. (2010) mostraram que derivados de petróleo, como gasolina e óleo, apresentam os valores da razão ${ }^{206} \mathrm{~Pb} /{ }^{207} \mathrm{~Pb}$ en- tre 1,13 e 1,15. Assim, um recente aporte de chumbo pode ter ocasionado mudança na composição isotópica desse metal no sedimento, o que não teria ocorrido nos oligoquetas, provavelmente por diferentes fatores: a) expostos a concentrações extremamente elevadas de chumbo, uma camada externa de muco secretado pelos oligoquetas adsorve quantidades significativas de chumbo e ferro, e serve como uma barreira para a entrada destes metais no organismo (Fleming \& Richards, 1982); b) submetidos a taxas elevadas de metais (cobre, ferro, chumbo e zinco), os oligoquetas aquáticos regulam o nível de metais em seu tecido (Bindra \& Hall, 1978); c) a existência de um intervalo de tempo entre a ingestão de sedimentos ricos em metais pesados e sua acumulação nos tecidos animais (Kennish, 1987).

Contudo, o fato dos valores da razão ${ }^{207} \mathrm{~Pb} /{ }^{206} \mathrm{~Pb}$ dos oligoquetas do canal do Una $(\sim 1,183)$ não serem similares àquele dos sedimentos de seu habitat $(1,167)$ não inviabiliza o uso desses organismos como bioindicadores, porque, mesmo parcialmente, o efeito da contribuição antropogênica foi registrado por eles.

\section{Conclusão}

A avaliação dos oligoquetas e poliquetas ( $N a-$ malycastis abiuma) como bioindicadores, através da composição isotópica do chumbo, permitiu identificar que esses organismos possuem comportamentos semelhantes. 0 fato de $75 \%$ dos organismos mostrarem associação positiva com os sedimentos permite dizer que tanto os oligoquetas quanto os exemplares N. abiuma podem registrar a assinatura isotópica do chumbo em seu habitat. Embora o número de amostras e análises nesse estudo tenha sido reduzido, os dados confirmam a hipótese de que esses organismos são bons bioindicadores e podem ser aplicados como uma matriz em estudos ambientais com isótopos de chumbo. Por outro lado, essa pesquisa aponta para a necessidade de um estudo mais detalhado sobre o processo biogeoquímico de assimilação e tempo de absorção metabólica de chumbo por parte dos oligoquetas.

\section{Referências}

Aviz, D.E, Carvalho, I.L.R., Rosa Filho, J.S. 2012. Spatial and temporal changes in macrobenthic communities in the Amazon coastal zone (Guajará Estuary, Brazil) caused by discharge of urban effluents. Scientia Marina, 76: 381390.

Azevedo, F.A. \& Chasin, A.A.M. 2003. Metais: gerenciamento da Toxicidade. São Paulo, Ed. Atheneu. p. 353-398.

Bindra, K.S. \& Hall, K.J. 1978. Bioaccumulation of selected trace metals by in benthic invertebrates in laboratory biossays. Relatório inédito preparado para NRC, Ottawa, 25p. (contrato-032-1082/6073).

Carigan J. E \& Gapiéry, C. 1995. Isotopic composition of epiphytic lichens as a tracer of the sources of atmospheric lead emissions in southern. Quebec, Canada. Geochimica et Cosmochimica Acta, 59: 4427-4433. 
Carvalho, C.M. 2001. Investigação do Registro Histórico da Composição Isotópica do Chumbo e da Concentração de Metais Pesados em Testemunhos de Sedimentos no Lago Água Preta, Região Metropolitana de Belém - Pará. Belém, 84p. Dissertação de Mestrado, Programa de Pós-graduação em Geociências, Instituto de Geociências, Universidade Federal do Pará.

Chaudhary-Webb, M. Paschal, D.C., Romieu, I., Ting, B, Elliot, C., Hopkins, H., Sanín, L.H. \& Ghazi, M.A. 2003. Determination lead sources in México using the lead isotope ratio. Salud Pública de México,v.45(2.):183-188. Disponível em: http://www.scielosp.org/pdf/spm/v45s2/v45s2a03. pdf. Acesso em: 19 jul. 2007.

Clark, R.B. 2001.Marine Pollution. New York, Oxford University Press, p. 117-118.

COHAB. Cooperativa Habitacional. 1997. Levantamento do quadro ambiental da zona metropolitana de Belém-produto 3, Belém. PNUB/IPESP/FADESP/COHAB, 158p.

Corrêa, J.A.M. \& Pereira, K.R. 2002. Estudo da dispersão de metais pesados em sedimentos da orla de Belém-PA. Boletim Museu Paraense Emílio Goeldi, Série Ciências da Terra, 14: 79-101.

Fauchald, K. \& Jumars. 1979. The Diet of Worms: a Study of Polichaete Feeding Guilds. Marine Biology Annual Review, 17:193-284.

Fleming, T.P. \& Richards K.S. 1982. Localization of Adsorbed Heavy Metals on the Earthworm Body Surface and their Retrieval by Chelation. Pedobiologia, 23:415-418.

Giere, 0. \& Pfannkuche, 0. 1982. Biology and ecology of marine Oligochaeta, a review. Oceanography and Marine Biology Annual Review, 20:173-308.

Gioia, S.M.C.L., Pimentel, M.M., Tessler, M.M, Dantas, E.L., Guimarães, E.M., Campos, J.E.G, Maruoka, M.T.S \& Nascimento, E.L.C. 2006. Sources of Anthropogenic Lead in Sediments From an Artificial Lake in Brasília - Central Brazil. Science of the Total Environment, 356:125-142.

Gray, J.S., McIntyre A.D. \& Stirn, J. 1992. Manual of methods in aquatic environment research. Part 11: Biological assessment of marine pollution - with particular reference to benthos. FAO Fisheries Technical Paper, 324, 49 p.

Gregório, A.M.S \& Mendes, A.C. 2009. Characterization of sedimentary deposits at the confluence of two tributaries of the Para River estuary (Guajara Bay, Amazon). Continental Shelf Research, 29(3):609-618.

IBGE. Instituto Brasileiro de Geografia e Estatística. 2008. Pesquisa Nacional de Saneamento Básico. Brasil, Brasília. Disponivel em: <http://www.ibge.gov.br/home/estatistica/populacao/ condicaodevida/pnsb2008/default. shtm>Acessado em: 05 out. 2010.

Kennish, M.J. 1987. Practical handbook of estuarine and marine pollution. Boca CRC Press LLC, Florida, 524p.

Langston, W.J. 1986. Metal in Sediments and Benhic Organisms in the Mersey Estuary. Estuarine, Coastal and Shelf Science, 23: 239-261.

Lima, C. S., Moura, C.A.V., Siqueira, N. V. M. \& Galarza, M. A. 2010. Determination of the isotope composition of lead and strontium in crude oil and derivatives and prospective environmental applications. In: SOUTH AMERICAN SYMPOSIUM ON ISOTOPE GEOLOGY, 7, 2010, Brasília. SSIG, V.7.

Manuscrito 489

Editores: Maria do Carmo Lime e Cunha e Paulo A. Souza.
Lima, W. N. \& Santos, M.T.P. 2001. Avaliação geoquímica ambiental de águas residuárias e de matéria orgânica degradada de canais de drenagem urbana (Belém, PA). Boletim do Museu Paraense Emílio Goeldi, Série Ciências da Terra, 13:3-40.

Moraes, B.C., Costa, J.M.N; Costa, A.C.L. \& Costa, M.H. 2005. Variação espacial e temporal da precipitação no Estado do Pará. Acta Amazonica, 35: 207-214.

Moura, C.A.V, Gaudette, H.E., Carvalho, M.C. \& Morales, G.P. 2004. The use of lead isotope composition as a tool to investigate the anthropogenic impacts on the environment in the metropolitan region of Belém (PA). Terrae, 1(1):8-15.

Nascimento, F.S. 1995. Dinâmica da Distribuição dos Poluentes Metálicos e Orgânicos nos Sedimentos de Fundo dos Canais de Drenagem de Belém, PA. Belém, 127p. Dissertação de Mestrado, Programa de Pós-graduação em Geociências, Instituto de Geociências, Universidade Federal do Pará.

Nascimento, S.M. 2007. Caracterização da assinatura isotópica do $\mathrm{Pb}$ atual e da concentração de metais pesados em sedimentos de fundo da foz do rio Guamá e da baía do Guajará, Belém-PA. Belém, 104p. Dissertação de Mestrado, Programa de Pós-graduação em Geociências, Instituto de Geociências, Universidade Federal do Pará.

Notten, M.J.M., Walraven, N., Beets, C.J., Vroon, P., Rozema, J. \& Aerts, R. 2008. Investigating the origin of $\mathrm{Pb}$ pollution in a terrestrial soil-plant-snail food chain by means of $\mathrm{Pb}$ isotope ratios. Applied Geochemistry, 23:1581-1593.

Pinheiro, R.V.L. 1987. Estudo hidrodinâmico e sedimentológico do estuário Guajará-Belém (PA). Belém, 164p. Dissertação de Mestrado, Programa de Pós-graduação em Geociências, Instituto de Geociências, Universidade Federal do Pará.

Pullianen, E., Lajunen, L.H.J. \& Itamies, J. 1986. Lead and Cadimium in Earthworms (Oligochaeta, Lumbricidae) in northern Finland. Annales Zoologici Fennici, 23: 303-306.

Ramos, J.F.F. 2004. Poluição e contaminação da Orla de Belém-PA. In: Uhly, S. \& E. L. Souza (eds.) A questão da água na grande Belém, pp. 121-148. Casas de Estudos Germânicos da UFPA, Belém.

Rodrigues, E.M.S. 1992. Implantação da metodologia $\mathrm{Pb}-\mathrm{Pb}$ em rocha total: exemplos de aplicação na Província Mineral de Carajás (PA). Belém, 128 f. Dissertação de Mestrado, Programa de Pós-graduação em Geociências, Instituto de Geociências, Universidade Federal do Pará.

Scheuhammer, A.M., Bond, D.E., Burgess, N.M. \& Rodrigues, J. 2003. Lead and stable lead isotope ratios in soil, earthworms, and bones of American woodcock (Scolopax minor) from Eastern Canada. Environmental Toxicology and Chemistry, 22 (11):2585-2591.

Singh, R.K., Chavan, S.L. \& Sapkale, P.H. 2007. Heavy Metal Concentrations in Water, Sediments and Body Tissues of Red Worm (Tubifex spp.) Collected from Natural Habitats in Mumbai, India. Environmental Monitoring and Assessment,,129:471-481.

Viana, A.P., Frédou, F.L.; Frédou, T \& Bordalo, A.O. 2009. Fish fauna as an indicator of environmental quality in a metropolitan region of the Amazon estuary. Journal of Fish Biology, 75:21-30. 